Supplement of Biogeosciences, 15, 2781-2801, 2018

https://doi.org/10.5194/bg-15-2781-2018-supplement

(C) Author(s) 2018. This work is distributed under

the Creative Commons Attribution 4.0 License.

(c) (1)

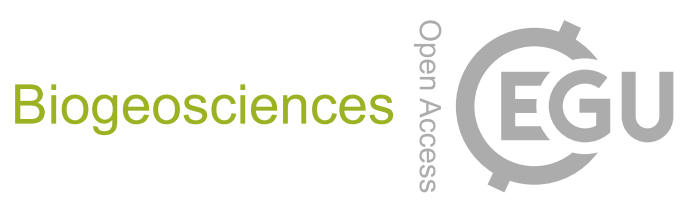

Supplement of

\title{
Spatial variation and linkages of soil and vegetation in the Siberian Arctic tundra - coupling field observations with remote sensing data
}

Juha Mikola et al.

Correspondence to: Mika Aurela (mika.aurela@fmi.fi)

The copyright of individual parts of the supplement might differ from the CC BY 4.0 License. 

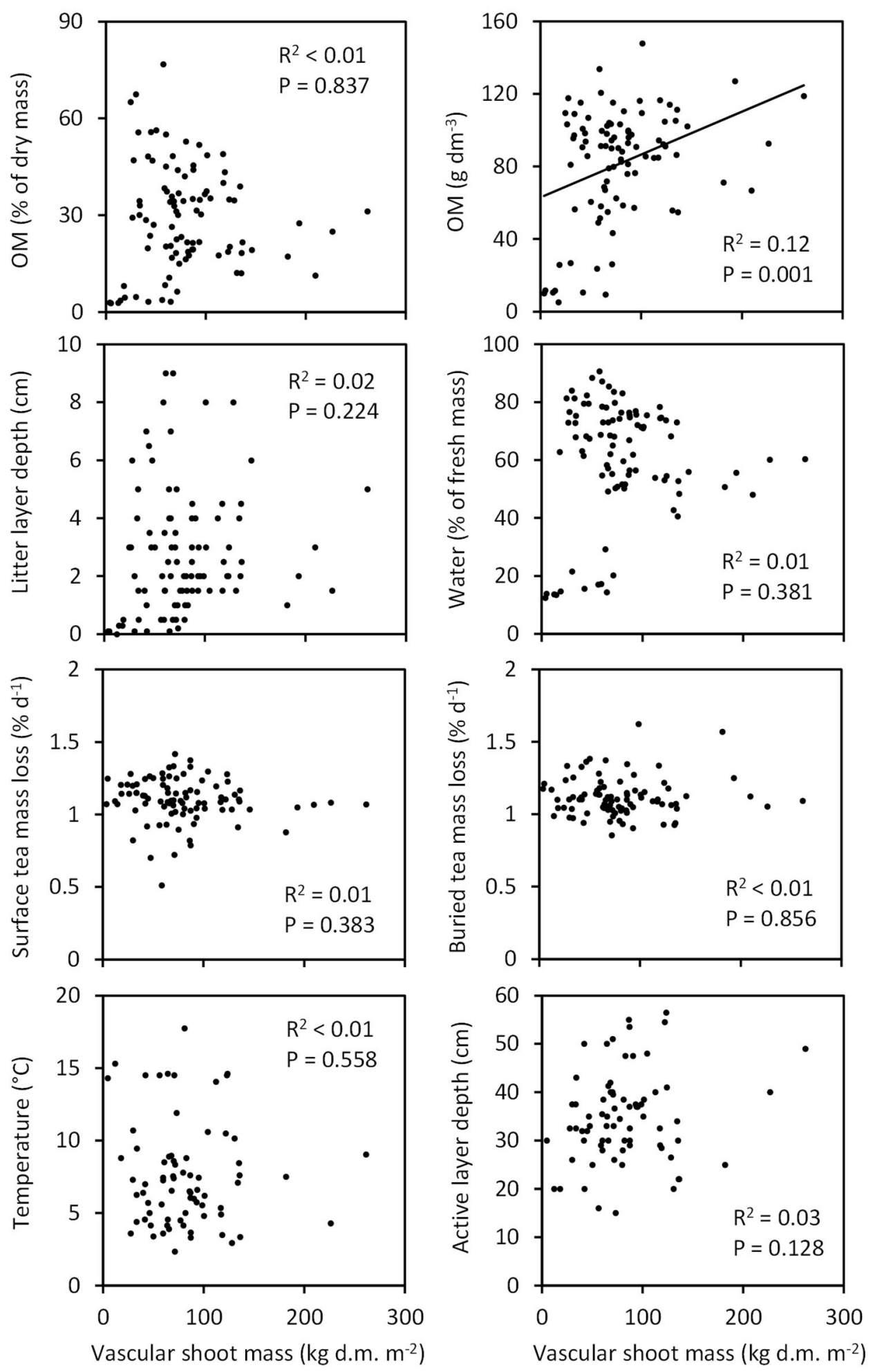

Supplementary Figure 1. Associations of vascular shoot biomass with soil characteristics across the Tiksi tundra field plots (see Fig. 10 for an explanation of the data and graphs). 
Supplementary Table 1. Number of study plots and mean values of the main soil and plant attributes in different land cover types. Carbon content is approximately $42 \%$ for moss dry mass, $45 \%$ for graminoid and herb dry mass, $50 \%$ for woody shrub dry mass and $58 \%$ for soil OM (Hobbie, 1996; Lal, 2004).

\begin{tabular}{|c|c|c|c|c|c|c|c|c|}
\hline & Bare soil & Lichen tundra & Shrub tundra & Flood meadow & Graminoid tundra & Bog & Dry Fen & Wet fen \\
\hline$n$ & 3 & 6 & 19 & 10 & 16 & 11 & 21 & 6 \\
\hline Soil OM concentration ( $\%$ of dry mass) & 2.8 & 4.9 & 20 & 22 & 33 & 40 & 42 & 37 \\
\hline Soil bulk density $\left(\mathrm{kg} \mathrm{dm}^{-3}\right)$ & 1.44 & 1.20 & 0.61 & 0.45 & 0.34 & 0.30 & 0.24 & 0.24 \\
\hline Soil OM content $\left(\mathrm{g} \mathrm{dm}^{-3}\right)$ & 11 & 17 & 76 & 89 & 97 & 98 & 93 & 79 \\
\hline Litter layer depth $(\mathrm{cm})$ & 0.07 & 0.27 & 2.1 & 2.0 & 2.8 & 4.9 & 3.6 & 2.8 \\
\hline Soil $\mathrm{pH}$ & 6.0 & 5.8 & 5.6 & 5.6 & 5.5 & 5.2 & 5.4 & 5.7 \\
\hline Soil water concentration ( $\%$ of fresh mass) & 13 & 25 & 47 & 57 & 70 & 70 & 76 & 77 \\
\hline Total moss dry mass $\left(\mathrm{g} \mathrm{m}^{-2}\right)$ & 0 & 57 & 280 & 238 & 523 & 820 & 674 & 86 \\
\hline Sphagnum dry mass $\left(\mathrm{g} \mathrm{m}^{-2}\right)$ & 0 & 0 & 0 & 6 & 265 & 391 & 309 & 23 \\
\hline Other moss dry mass $\left(\mathrm{g} \mathrm{m}^{-2}\right)$ & 0 & 57 & 280 & 232 & 258 & 429 & 365 & 63 \\
\hline Total vascular plant dry mass $\left(\mathrm{g} \mathrm{m}^{-2}\right)$ & 7 & 47 & 112 & 88 & 78 & 93 & 58 & 86 \\
\hline Betula nana dry mass $\left(\mathrm{g} \mathrm{m}^{-2}\right)$ & 0 & 3.6 & 29 & 1.2 & 0.2 & 23 & 5.8 & 0 \\
\hline Dwarf shrub dry mass $\left(\mathrm{g} \mathrm{m}^{-2}\right)$ & 3.3 & 7.0 & 48 & 0 & 0 & 35 & 1.1 & 0 \\
\hline Salix dry mass $\left(\mathrm{g} \mathrm{m}^{-2}\right)$ & 0 & 3.3 & 11 & 16 & 35 & 23 & 15 & 2.0 \\
\hline Herb dry mass $\left(\mathrm{g} \mathrm{m}^{-2}\right)$ & 2.5 & 31 & 17 & 14 & 2.6 & 1.1 & 4.1 & 1.7 \\
\hline Graminoid dry mass $\left(\mathrm{g} \mathrm{m}^{-2}\right)$ & 1.0 & 1.8 & 6.7 & 56 & 40 & 11 & 32 & 82 \\
\hline Vascular plant LAI $\left(\mathrm{m}^{2} \mathrm{~m}^{-2}\right)$ & 0.04 & 0.20 & 0.51 & 0.95 & 0.62 & 0.42 & 0.44 & 0.85 \\
\hline$n$ & 2 & 11 & 14 & 11 & 15 & 14 & 15 & 8 \\
\hline Early July (160 DD) soil T $\left({ }^{\circ} \mathrm{C}\right)$ at $15 \mathrm{~cm}$ depth & 6.7 & 6.6 & 2.5 & 2.6 & 1.7 & 1.9 & 1.8 & 3.1 \\
\hline Early August (380 DD) soil T $\left({ }^{\circ} \mathrm{C}\right)$ at $15 \mathrm{~cm}$ depth & 15 & 13 & 6.9 & 9.1 & 6.8 & 5.2 & 6.3 & 8.8 \\
\hline Early July (160 DD) active soil layer depth (cm) & 24 & 24 & 14 & 22 & 16 & 15 & 16 & 24 \\
\hline Mid-August (550 DD) active soil layer depth (cm) & 26 & 25 & 28 & 41 & 35 & 35 & 35 & 43 \\
\hline
\end{tabular}


Supplementary Table 2. Accuracy assessment of the land cover classification (values in the table refer to $2 \mathrm{~m}$ resolution pixels).

\begin{tabular}{|c|c|c|c|c|c|c|c|c|c|c|c|c|}
\hline \multirow{13}{*}{$\begin{array}{c}\text { Predicted } \\
\text { data }\end{array}$} & \multicolumn{12}{|c|}{ Reference data } \\
\hline & & Dry fen & Wet Fen & Bog & $\begin{array}{l}\text { Lichen } \\
\text { tundra }\end{array}$ & $\begin{array}{l}\text { Shrub } \\
\text { tundra }\end{array}$ & $\begin{array}{l}\text { Graminoid } \\
\text { tundra }\end{array}$ & $\begin{array}{l}\text { Flood } \\
\text { meadow }\end{array}$ & $\begin{array}{l}\text { Bare } \\
\text { soil }\end{array}$ & Water & $\begin{array}{l}\text { Total } \\
\text { predicted }\end{array}$ & $\begin{array}{l}\text { User's } \\
\text { accuracy }\end{array}$ \\
\hline & Dry fen & 1202 & 129 & 316 & 0 & 350 & 685 & 70 & 0 & 0 & 2752 & $44 \%$ \\
\hline & Wet Fen & 593 & 1073 & 288 & 8 & 303 & 410 & 203 & 0 & 0 & 2878 & $37 \%$ \\
\hline & Bog & 325 & 125 & 1094 & 34 & 309 & 388 & 176 & 0 & 0 & 2451 & $45 \%$ \\
\hline & Lichen tundra & 55 & 0 & 0 & 1072 & 464 & 75 & 37 & 213 & 0 & 1916 & $56 \%$ \\
\hline & Shrub tundra & 451 & 31 & 316 & 135 & 1484 & 367 & 176 & 0 & 0 & 2960 & $50 \%$ \\
\hline & Graminoid tundra & 367 & 217 & 103 & 0 & 64 & 965 & 469 & 0 & 0 & 2185 & $44 \%$ \\
\hline & Flood meadow & 0 & 0 & 0 & 14 & 62 & 0 & 135 & 33 & 0 & 244 & $55 \%$ \\
\hline & Bare Soil & 0 & 0 & 0 & 571 & 0 & 0 & 98 & 929 & 0 & 1598 & $58 \%$ \\
\hline & Water & 0 & 0 & 0 & 0 & 15 & 0 & 48 & 83 & 858 & 1004 & $86 \%$ \\
\hline & Total in reference & 2993 & 1575 & 2117 & 1834 & 3051 & 2890 & 1412 & 1258 & 858 & & \\
\hline & Producer's accuracy & $40 \%$ & $68 \%$ & $52 \%$ & $59 \%$ & $49 \%$ & $33 \%$ & $10 \%$ & $74 \%$ & $100 \%$ & & \\
\hline
\end{tabular}

